\title{
Cylindrical Cam Profile by CMM Based on Pro/E Model
}

\author{
Chao Ma ${ }^{1}$, a , Bin Wang ${ }^{1}$, Liu Xu' ${ }^{1}$, Yan $\mathrm{Yu}^{1}$, and Ruolei $\mathrm{Li}^{1}$ \\ ${ }^{1}$ Military Transportation University, Tianjin 300161, China \\ amachaobbnc@vip.qq.com
}

\begin{abstract}
Keywords: Cylindrical Cam;Pro/E Model;CMM
Abstract. The development of CAD and CAM technology makes it more process to inspect surfaces of the part, this is usually carried out by CMM. With the research of the error analysis of cutter location for unequal diameter side milling of cylindrical cam, the technology using CMM to inspect surfaces by Pro/E CAD model is analysed from some key aspects: Pro/E modeling; set up coordinate ; and probe compensation.
\end{abstract}

\section{Introduction}

Precision measurement technology is the mechanical industrial development foundation and technological conditions.Precision measurement and control is one pillar of modern precision engineering. For science and technology, precision measurement and control engineering is development promoting factor. Measurement accuracy and efficiency decide the level of processing. In recent years, CMM application is rapidly developing in manufacturing industry,and it become standard equipment of 3D detection industrial. CMM solved the measurement of complex surface, such as blade ,automobile engine hood and aircraft wing[1].

\section{Cylindrical Cam Processing Method}

According to relationship between the radius of tool and the radius of cylindrical cam roller , Cylindrical cam processing methods can be divided into equal diameter method and unequal diameter method. Equal diameter method is the method that the radius of tool is consistent with the radius of cylindrical cam roller, otherwise known as the unequal diameter method.

The purpose of this study is explore new unequal diameter machining cylindrical cam method in the range of allowable error. However, cylindrical cam surface is not developable surface,cylindrical cam machining is a technical problem [2].

The experiment with the double envelope principle is showed in Fig.1. The radius of cylindrical cam roller is $r_{0}$, the radius of unequal diameter tool is $r_{c}$, and $r_{0}<r_{c}$. The unequal diameter tool is deviated from the roller axis for $\Delta r=r_{0}-r_{c}$, and turn around of the roller axis of rotation. The envelope is equal to the radius of cylindrical $S_{1}$, then $S_{1}$ moves along cylindrical cam movement, $S 2$ is cylindrical cam surface [3].

\section{The Three Coordinate Measuring of Cylindrical CAM Profile}

Cylindrical Cam Specimen. This test select the diameter for $\phi 16 \mathrm{~mm}$, ф $22 \mathrm{~mm}$ and $\phi 25 \mathrm{~mm}$ three kind of milling cutter. Test cylindrical CAM actual profile surface by diameter for $\phi 25 \mathrm{~mm}$ column follower envelope and become, cylindrical CAM outside diameter for $\phi 150 \mathrm{~mm}$, inner diameter for ф $120 \mathrm{~mm}$.

In order to reduce the clamping error, this test in cylindrical blank face of respectively on using the above three kinds of the diameter of the tool, every 120 degrees processing a 100 degree convex contour surface. In addition, in order to facilitate the three coordinate measuring machine gauge head acquisition data, test will diameter for $\phi 150 \mathrm{~mm}$ of specimen processing a $\phi 120 \mathrm{~mm}$ through holes. Cylindrical CAM model as shown in Fig.1 shows. 


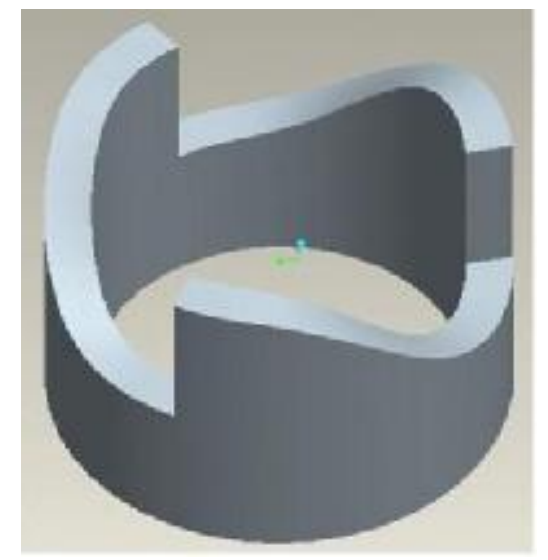

Fig.1 Cylindrical Cam Model

The Establishment of Cylindrical CAM model by Pro/E. Pro/E based on the characteristics of the parametric modeling function in establishing the process of cylindrical CAM model plays an important role. Due to the cylindrical CAM contour surface and roller contact line of the machining accuracy is not equivalent to measure the quality of processing the important index, so contact line of some contact point is to establish a geometric model and three coordinate measuring key.

Modeling process screenshots as shown in Fig.2. In order to guarantee cylindrical CAM geometric model is correct into three coordinates measuring machine measuring software, the completion after modeling in Pro/E software will PRT format model file save as IGES data files as three coordinates measuring machine of the input file.
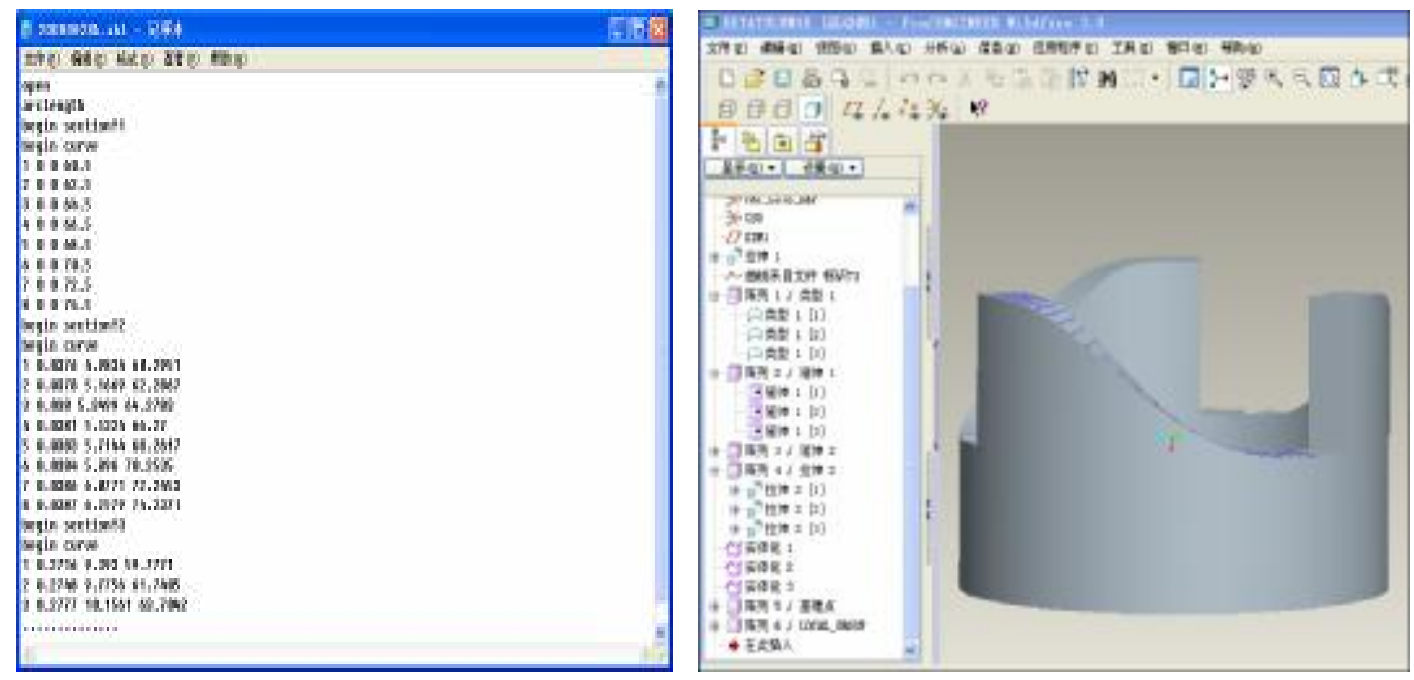

Fig.2 Model Process Print Screen

Measuring equipment of Cylindrical CAM Profile. This test and measurement equipment used by the Spanish Trimek company produces the SPARK10.07.07 type three coordinate measuring machine. By the test piece with graphic representation in the main interface, measuring path advance display greatly shorten the measuring time.

Equipment main parameters: the Metrology - XP numerical control system, the unit pulse equivalent to $0.0005 \mathrm{~mm}$ and $7.5 \mathrm{deg}$. Stroke parameters: $\mathrm{X}$ axis: $1000 \mathrm{~mm}, \mathrm{Y}$ axis: $700 \mathrm{~mm}, \mathrm{Z}$ axis: $700 \mathrm{~mm}$, A shaft: $360 \mathrm{deg}$, B axis: $105 \mathrm{deg}$. Auxiliary parameters: the fastest speed $200 \mathrm{~mm} / \mathrm{SEC}$, positioning accuracy $3+31 / 1000 \mu \mathrm{m}$ maximum specimen weight $1900 \mathrm{~kg}$. Measuring head for RENISHAW contact degree of freedom - PH110T type, measuring head repeated positioning accuracy for $5 \mu \mathrm{m}$. 


\section{Key Problem of Measuring}

The Establishment of CMM Specimen Coordinate System. In the use of three coordinates measuring machine to specimen testing before, must first build corresponding measuring coordinate system, its directly affect the measurement accuracy.

This test used the 321 method to establish the workpiece coordinate system [3]. This test coordinate system to establish process shows in Fig.3:

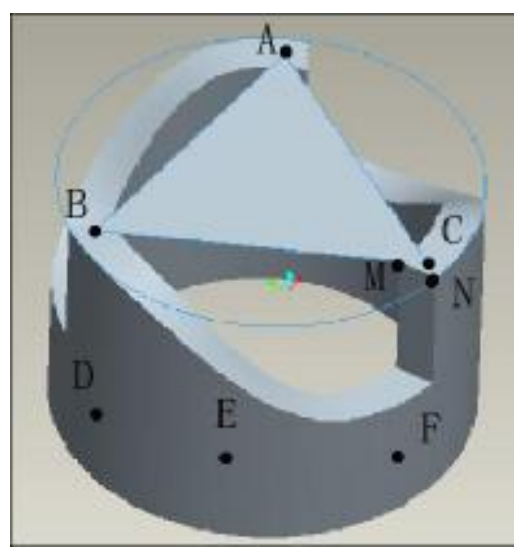

Fig.3 the Model of Coordinate of the Cylindrical Cam

In end face datum plane was determined through A, B and C. Datum plane normal is considered as the first axis directionof specimen coordinate system.

In the $\mathrm{ABC}$ three sure not fair face select edge $\mathrm{MN}$ as to be built specimen coordinate system the second axis direction.

Due to the specimen is cylindrical CAM, so in cylindrical cylindrical on selection in $3 \mathrm{D}, \mathrm{E}, \mathrm{F}$, determine the cylindrical profile, the center of circle set to to be built coordinate system origin.

Compensation of Tip Radius. At present, three coordinate measuring machine widely used mechanical trigger type probe, when measuring head contact to the specimen, three coordinate measuring machine receive coordinate values should be nose center coordinates, when the surface of the measuring method of vector direction and the measuring of the axis direction consistent, shows in Fig.4. Measuring head along the axial direction measurement back, measuring software will automatically compensate a nose radius as the measured value. For plane, round and standard features, can through the whole biased automatic compensation measured value, for continuous scanning curve, also can use the same way to be automatic place.

But for measuring free surface, measurement axis and the measuring point surface method vector direction not collinear. Mining point began, measuring software will along the axis of measuring the direction of radius compensation. Be compensation point is not the real contact point $\mathrm{P}$, but along the measuring head axis direction of the extension line on a point $\mathrm{Q}$, thus caused the compensating error. The size of the errors and measuring head radius $r$ and the specimen measured surface and cartesian coordinate axis Angle are concerned, the greater the Angle, the greater the error. Compensating error and the measuring head radius $r$ proportional relations, namely measuring head radius $r$ is smaller, and the measurement error of the small. So when three coordinates measuring machine in standard point measurement, should choose as far as possible little nose. When measuring head axis and measured the surface normals Angle between $\mathrm{a}=0$, measuring head radius compensation error also is 0 . So the measurement will be as far as possible measuring head axis and the measured surface vertical, make the measuring head along the measured surface normal direction, in order to maximize reduce measuring head radius compensation error . 


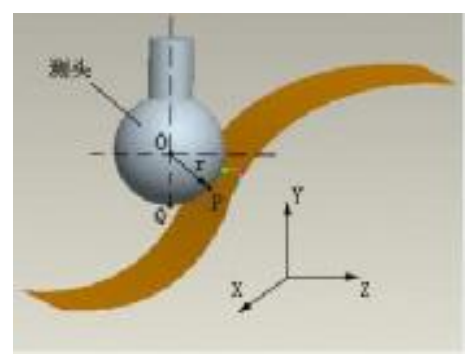

Fig.4 Compensation of Tip Radius

\section{Conclusion}

Based on Pro/E 3 d geometric model of three-dimensional cylindrical CAM contour surface measurement technology belong to a comprehensive strong measurement technology. In this measuring cylindrical CAM contour surface process, reasonable design of CAM specimen full consideration of gauge head of the data gathering convenience and measuring head radius compensation accuracy, in which maximum except loading entrainment to error. Three coordinate measuring cylindrical CAM contour surface, can be used for analysis, research nonequivalence machining cylindrical CAM contour surface error.

\section{References}

[1] R.S.Lee and J.N.Lee . a New Tool-Path Generation Method Using a Cylindrical End Mill for 5-Axis Machining of a Spatial Cam with a Conical Meshing Element.Int.J.Adv.Manuf Tech nol 2001,18:615-623.

[2] Ma Chao, Niu Xinghua, Zhang Zhe: Journal of Tianjin University of Technology, Vol.26 (2010) No.3, p27-29.

[3] Guo Wei Zhong: Journal of China Three Gorges University(Natural Sciences), Vol.23 (2001) No.2, p159-161. 